\title{
Vanin-1/- mice show decreased NSAID- and Schistosoma- induced intestinal inflammation associated with higher glutathione stores
}

\author{
Florent Martin, ${ }^{1}$ Marie-France Penet, ${ }^{2}$ Fabrice Malergue, ${ }^{1}$ Hubert Lepidi, ${ }^{3}$ Alain Dessein, ${ }^{2}$ \\ Franck Galland, ${ }^{1}$ Max de Reggi, ${ }^{2}$ Philippe Naquet, ${ }^{1}$ and Bouchra Gharib ${ }^{2}$ \\ ${ }^{1}$ Centre d'Immunologie de Marseille-Luminy, Centre National de la Recherche Scientifique-Institut National de la Santé \\ et de la Recherche Médicale, \\ ${ }^{2}$ Institut National de la Santé et de la Recherche Médicale U 399, and \\ ${ }^{3}$ Unité des Rickettsies-Centre National de la Recherche Scientifique, UMR 6020, Université de la Méditerranée, \\ Marseille, France
}

\begin{abstract}
Vanin-1 is a membrane-anchored pantetheinase highly expressed in the gut and liver. It hydrolyzes pantetheine to pantothenic acid (vitamin B5) and the low-molecular-weight thiol cysteamine. The latter is believed to be a key regulating factor of several essential metabolic pathways, acting through sulfhydryl-disulfide exchange reactions between sulfhydryl groups of the enzymes and the oxidized form, cystamine. Its physiological importance remains to be elucidated, however. To explore this point, we developed Vanin-1-deficient mice that lack free cysteamine. We examined the susceptibility of deficient mice to intestinal inflammation, either acute (NSAID administration) or chronic (Schistosoma infection). We found that Vanin-1/- mice better controlled inflammatory reaction and intestinal injury in both experiments. This protection was associated with increased $\gamma$-glutamylcysteine synthetase activity and increased stores of reduced glutathione, as well as reduced inflammatory cell activation in inflamed tissues. Oral administration of cystamine reversed all aspects of the deficient phenotype. These findings suggest that one cysteamine function is to upregulate inflammation. Consequently, the pantetheinase activity of Vanin-1 molecule could be a target for a new anti-inflammatory strategy.

J. Clin. Invest. 113:591-597 (2004). doi:10.1172/JCI200419557.
\end{abstract}

\section{Introduction}

Vanin genes were discovered recently following mouse thymus reconstitution experiments after irradiation (1). The mouse gene cluster includes Vanin-1 and Vanin-3 $(2,3)$ preferentially expressed by epithelial and myeloid cells, respectively (4). Vanin-1 and Vanin-3 transcripts show ubiquitous but heterogeneous tissue distribution in vivo, with Vanin-1 being the predominantly expressed isoform in the gut. Vanin-1 is a glycosylphosphatidyl inositol (GPI) anchored to cell membranes, whereas Vanin-3 is secreted. Vanin genes encode a pantetheinase (EC 3.5.1.) $(5,6)$ that hydrolyzes pantetheine to pantothenic acid (vitamin B5) and cys-

Received for publication July 22, 2003, and accepted in revised form December 16, 2003.

Address correspondence to: Bouchra Gharib, INSERM U 399, Faculté de médecine, 27, Boulevard Jean Moulin, 13385, Marseille Cedex 5, France. Phone: 33-491-32-44-54; Fax: 33-491-79-60-63; E-mail: bouchra.gharib@medecine.univ-mrs.fr.

Florent Martin and Marie-France Penet contributed equally to this work.

Philippe Naquet and Bouchra Gharib are co-senior authors.

Conflict of interest: The authors have declared that no conflict of interest exists.

Nonstandard abbreviations used: glycosylphosphatidyl inositol (GPI); $\gamma$-glutamylcysteine synthetase ( $\gamma$-GCS); glutathione (GSH); hemoglobin (Hb); cyanin-3 (Cy3); epithelial cell adhesion molecule (Ep-CAM); myeloperoxidase (MPO); cyclooxygenase-1 (COX-1); macrophage inhibitory protein-2 (MIP-2); hypoxanthine phosphoribosyl transferase (HPRT); GSH reductase (GR). teamine (7). Cysteamine is a low-molecular thiol broadly distributed in organisms, from Drosophila to humans. This wide distribution and the mechanisms involving low-molecular thiols are likely to reflect the early steps of molecular evolution (8). In vivo, cysteamine is in equilibrium with its oxidized form cystamine at concentrations of approximately $20 \mu \mathrm{M}$ (9-12). Cystamine is believed to be a key regulator of essential metabolic pathways. It acts by sulfhydryl-disulfide exchange reactions between sulfhydryl groups at or near the active site of the enzymes and the oxidized form, cystamine (13-16). The enzymes putatively inhibited by cysteamine include $\gamma$-glutamylcysteine synthetase $(\gamma$-GCS), which catalyzes the key step in glutathione (GSH) synthesis (17-19). This enzyme activity is of central importance because GSH fulfills many important and chemically complex roles in protecting cellular components from the deleterious effects of toxic species (20). Cysteamine can have dramatic effects, since its administration is widely used as an experimental model of duodenal ulcers (21). Nevertheless, the physiological importance of cysteamine remains elusive.

To explore this point, we developed Vanin-1-deficient (Vanin-1/-) mice. Deficient mice lack free cysteamine (6). Because of the high Vanin-1 gene expression in the gut and cysteamine-induced intestinal injury, we examined the susceptibility of Vanin-1/-- mice to NSAID intestinal injury. In addition, we used a model of chronic intestinal inflammation, Schistosoma infection. The Schistosoma 
model is extensively used to investigate liver delayed hypersensitivity response. In humans, the severity of hepatic injury associated with schistosomiasis is under the control of the 6q22-q23 locus, referred to as SM2 (22). This region is highly syntenic with mouse 10A2B1, the location of Vanin gene cluster. In this study, we investigated intestine injury following Schistosoma infection, because the intestine is the second main site of egg deposition injury (23). In both models, tissue damage is associated with oxidative stress. Mice that lack NADPH oxidase activity (gp91 phox $^{-/}$) are less susceptible to NSAID injury than WT mice (24). Similarly, schistosomiasis is characterized by an altered balance between pro-oxidant and antioxidant process inflammation (25-28).

We showed that Vanin-1 $1^{-/}$mice better controlled inflammatory reaction and intestinal injury in both experiments. This protection was associated with increased $\gamma$-GCS activity and GSH stores, as well as reduced inflammatory cell activation in inflamed tissues. Oral administration of cysteamine reversed all aspects of the deficient phenotype. These findings suggest that one of cysteamine's function is to upregulate inflammation. Consequently, the pantetheinase activity of the Vanin-1 molecule could be a target for a new anti-inflammatory strategy.

\section{Methods}

Mice. Vanin-1/- mice backcrossed on a BALB/c background (nine generations) were kept in a specific pathogen-free mouse facility and handled according to the rules of Décret n 87-848 du 19/10/1987, Paris. Animal experiments were performed according to the legal authorization certificates delivered for the Centre d'Immunologie de Marseille Luminy (authorization 007031) and one of the group leaders (P. Naquet, authorization 13-70).

Drug administration. Indomethacin (Sigma-Aldrich, St. Louis, Missouri, USA) was administered at a dose of 25 $\mathrm{mg} / \mathrm{kg}$ in $5 \%$ sodium carbonate to Vanin-1/- $(n=5)$ and WT $(n=5)$ mice in two subcutaneous injections at 12hour intervals. Mice were sacrificed 20 hours after the first injection. Cystamine (Sigma-Aldrich) was given by gavage three times on day 1 and once on day 2 at the moderate total dose of $120 \mathrm{mg} / \mathrm{kg}$. Mice were sacrificed 5 hours after the last gavage. To evaluate the impact of cystamine on indomethacin treatment (Sigma-Aldrich), Vanin-1-/- $(n=5)$ and WT $(n=5)$ mice received cystamine prior to indomethacin injection. Intestinal bleeding was evaluated by harvesting the whole intestine and disrupting the tissue in water. Serial dilutions were used to quantify hemoglobin $(\mathrm{Hg})$ content using Hemastix Reagent Strips (Bayer AG, Leverkusen, Germany). Results were expressed in milligrams of $\mathrm{Hb}$ per gram of tissue.

Infection with Schistosoma mansoni. Vanin-1 $1^{-/-}(n=10)$ and WT $(n=10)$ mice were percutaneously infected with 150 cercariae of Schistosoma mansoni (Puerto Rican strain). A first cohort was used for survival studies and three others for biological assays.
Survival studies. We followed survival of mice daily after S. mansoni infection. During the acute phase of the disease (8-12 weeks after infection) (29), the severity of intestinal injury was monitored by quantifying the presence of $\mathrm{Hb}$ in feces. For this purpose, droppings were collected daily and resuspended in a $1: 5 \mathrm{wt} / \mathrm{vol}$ ratio of distilled water. Samples were diluted ten times and the $\mathrm{Hb}$ concentration was assayed as done previously.

Histopathological analysis. After sacrifice, small intestines of the indomethacin-treated mice were removed, formalin fixed, and paraffin embedded. They were cut to $4-\mu \mathrm{m}$ thickness and stained with hematoxylin-phloxine-saffron. Immunohistology was performed on small intestine cryosections using the anti-Vanin-1 H202-407 mAb (1) revealed with a mouse-adsorbed goat anti-rat Ig-HRP (Southern Biotechnology Associates Inc., Birmingham, Alabama, USA) using tyramide-cyanin-3 (Cy3) as substrate (Perkin Elmer Life Sciences Inc., Boston, Massachusetts, USA). Villus height and width were measured on 15 sections per mouse (three WT and three Vanin-1/indomethacin-treated mice) obtained for every 500 $\mu \mathrm{m}$ of small intestine, using a quantitative image analysis system (Biocom Technologies, Poulsbo, Washington, USA).

Colons of Schistosoma-infected mice were frozen in OCT, cut to $10-\mu \mathrm{m}$ thickness, and stained with H\&E. Double-labeling studies were performed using a combination of FITC-coupled anti-epithelial cell adhesion molecule (anti-Ep-CAM) $\mathrm{mAb}(30)$ and direct incubation with the tyramide-Cy3 substrate for the detection of myeloid cells showing active peroxidase activity. Quantification of peroxidase-positive areas was performed using the Metamorph software (Universal Imaging Corp., Downingtown, Pennsylvania, USA) using pictures taken of distinct areas of tissues $(\times 10)$. Results were obtained from the analysis of four to ten different pictures per mouse (WT $=6$, Vanin- $1^{-/-}=8$ ), and the following information was gathered: total analyzed area in square micrometers, percentage of stained area, mean area of stained cells (one cell giving a signal of 20-50 $\mu \mathrm{m}^{2}$ ). Confocal microscopy analysis was performed similar sections stained with FITC-coupled anti-Ep-CAM mAb and tyramide-Cy5 using a Zeiss Confocor2 microscope (Carl Zeiss SAS, Le Pecq, France). Ten-micrometer optical sections were acquired on the whole thickness of the tissue.

Myeloperoxidase activity. Intestinal samples $(3 \mathrm{~cm})$ taken from the most injured part, that is, the colon of S. mansoni-infected mice and the small intestine of indomethacin-treated mice, were homogenized in $0.5 \%$ hexadecyltrimethyl-ammonium bromide. Myeloperoxidase (MPO) activity was determined as described previously (31).

Semiquantitative RT-PCR. Total cellular RNA was isolated from small intestine samples of indomethacintreated mice by guanidinium thiocyanate phenolchloroform extraction. The cDNA amplifications 

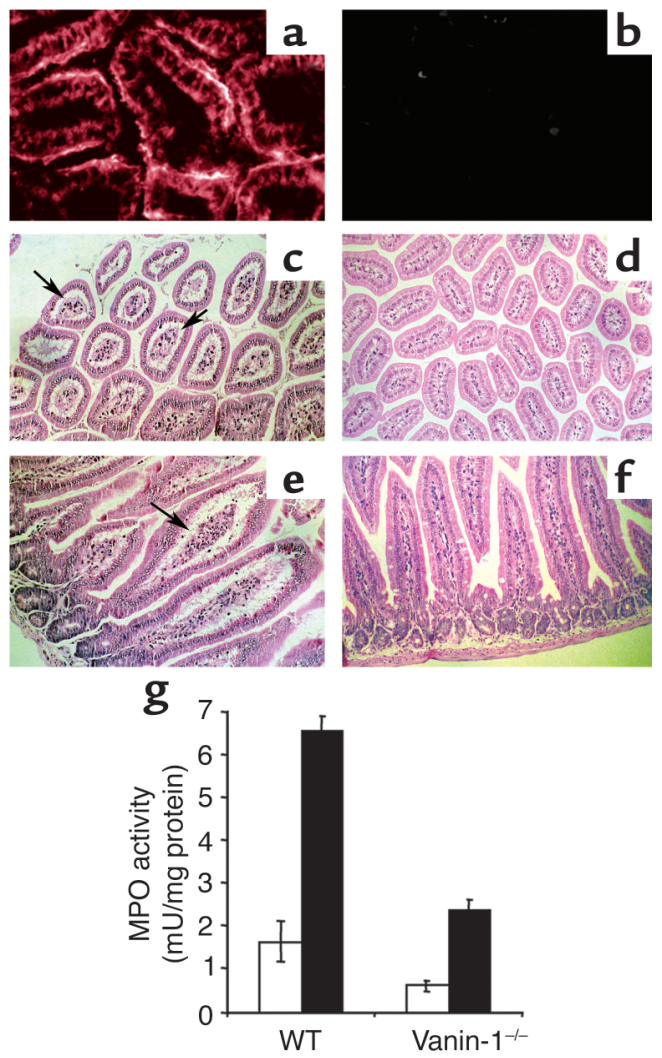

\section{Figure 1}

(a-f) Representative histological sections of small intestine in indomethacin-treated mice. Immunohistology of control WT (a) and Vanin-1/- (b) mice using anti-Vanin-1 mAb labeling. Cross and longitudinal intestinal sections from WT (c and $\mathbf{e})$ and Vanin-1-/( $\mathbf{d}$ and $\mathbf{f}$ ) mice; arrows, swollen intestinal villi and dilated lymphatic channels. Hematoxylin-phloxine-saffron staining. Magnification: $\times 160$ (a and b), $\times 40$ (c and d), and $\times 100$ (e and f). (g) Lower intestinal MPO activity in indomethacin-treated WT and Vanin-1/- mice (white bars, control; black bars, treated). Values are means \pm SD. Values from Vanin-1/-- and WT groups are significantly different $(P<0.05$, Student $t$ test $)$.

were performed using the following primer sets: iNOS (32), cyclooxygenase-1 (COX-1) (33), COX-2 (34), macrophage inhibitory protein-2 (MIP-2) (35), and hypoxanthine phosphoribosyl transferase (HPRT) (36). Relative quantification of iNOS and COX-2 gene expression was carried out as done previously (37). For each primer set, six PCR analyses were performed, ranging from 32 to 42 amplification cycles. Bands were scanned (The Imager; Appligene, Illkirch, France), and OD was quantitated using an image analyzer (Biocom Technologies). The results were plotted on a semilogarithmic scale against the sampling cycle number to obtain amplification curves. Values obtained at 36 cycles, which were within the linear phase for all sets, were related to HPRT values.

Determination of $\gamma$-GCS activity and GSH levels in liver and intestine. The $\gamma$-GCS activity was determined as described (38). GSH levels were determined according to Tietze et al. (39).
Statistical analysis. Data are expressed as mean plus or minus SD. Values from experimental and control groups were compared using the Student $t$ test. $P$ values less than 0.05 were considered statistically significant.

\section{Results}

Lack of Vanin-1 reduced intestinal injury following indomethacin treatment. Vanin-1 is highly expressed on enterocytes in WT but not in Vanin-1/- mice and is preferentially localized at the brush border (Figure 1, a-f). Histological examination of intestinal injury following indomethacin treatment showed that WT mice had altered intestinal villi, which appeared swollen and increased in width. At higher magnification, we noted an edema of the lamina propria under the epithelial lining of the intestinal villi. In contrast, the intestinal wall of the Vanin-1-1- mice appeared histologically normal, without architectural disorganization of the intestinal villi. Morphometric analysis showed shorter and larger villi in WT mice as compared with Vanin-1//mice (height, $407 \pm 34.6 \mu \mathrm{m}$ versus $528 \pm 35.7 \mu \mathrm{m}$; width, $132 \pm 12.4$ Ïm versus $98 \pm 7.9 \mu \mathrm{m}$, respectively). Consequently, the length/width ratio was drastically diminished in the WT mice (Student $t$ test; $P<0.001$ ). Furthermore, in several areas WT mice villi were severely damaged and disrupted (data not shown). These structural changes were associated with a drastic reduction of MPO activity (Figure $1 \mathrm{~g}$ ). Moreover, intestinal bleeding was observed in WT mice, with $9-21 \mathrm{mg} \mathrm{Hb} / \mathrm{g}$ tissue, in contrast to less than $2 \mathrm{mg}$ $\mathrm{Hb} / \mathrm{g}$ tissue in Vanin-1/- mice. Thus, the absence of Vanin-1 attenuates duodenal injury.

Lack of Vanin-1 reduced inflammatory response to indomethacin treatment. We evaluated the expression of a number of inflammatory factors following indomethacin treatment. Basal COX-1 mRNAs were expressed equally in WT and Vanin-1/- mice; under our experimental conditions, mRNA levels were not affected whatever treatment was applied. The other mRNAs assayed were expressed at low basal levels in control conditions (Figure 2). Indomethacin treatment triggered a sharp MIP-2 mRNA expression in WT but not in Vanin-1-/ mice, while iNOS and COX-2 mRNA levels were fourfold and twofold higher, respectively, in WT than in Vanin-1 $1^{-/}$mice. To link firmly the reduced inflammatory factor expression in Vanin-1/- mice to cyst(e)amine deficiency, cystamine was given to indomethacin-treated mice. The treatment reversed the Vanin $-1^{-/}$phenotype; that is, it restored mRNA expression to WT levels. The effect was not due to cystamine itself, because under our conditions cystamine administered alone did not change iNOS and MIP- 2 mRNAs despite a moderate upregulation of COX-2. The lack of detectable MIP-2 mRNA in the absence of Vanin-1 was in agreement with the drastic reduction of intestinal MPO (Figure 1g).

Vanin-1/- mice survived longer following S. mansoni infection. The severity of intestinal inflammation is a prognostic factor in the pathology induced by S. man- 


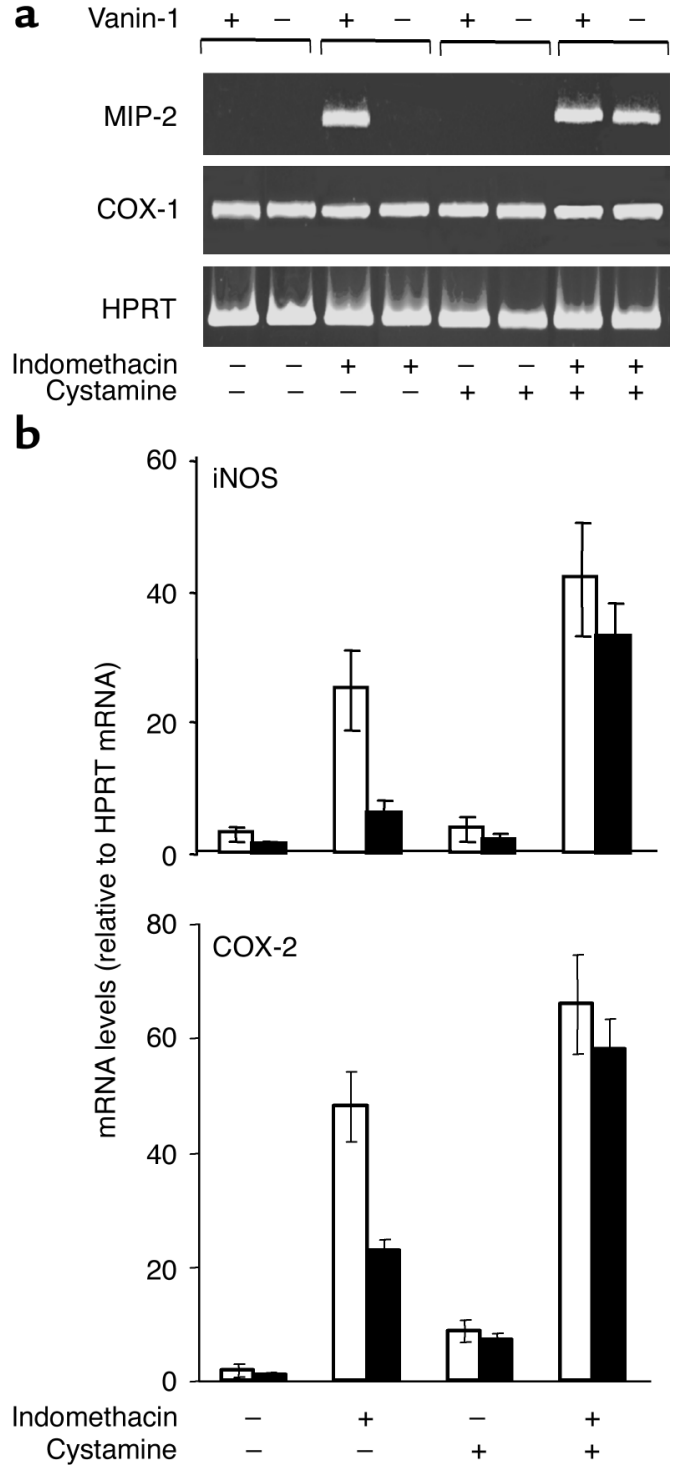

Figure 2

Reduced expression of inflammatory factors in Vanin-1/- mice. (a) Representative experiment of semiquantitative RT-PCR analysis of MIP-2 and COX-1 mRNAs in the small intestine of untreated or indomethacin-treated Vanin-1/- and WT mice. Cystamine was administered when indicated. Amplification of HPRT mRNA transcript was used as internal control. (b) Relative iNOS and COX-2 mRNA levels after PCR amplification.

soni infection (40). Indeed, the 8- to 12 -week postinfection period is the acute phase of $S$. mansoniinduced inflammation. This period was critical for survival of WT mice, with eight out of ten mice dying at this time. Mortality was delayed in the Vanin-1-/group, and only three out of ten mice died during this acute-phase period. Survival in the mutant group was then stable for 4 weeks, until deaths resumed at 16 weeks after infection (Figure 3a). After 20 weeks after infection, only two Vanin $-1^{-/}$mice were still alive. Among the eight $S$. mansoni-infected WT mice that died during the 8 - to 12 -week postinfection period, five showed severe intestinal bleeding, with a very high $\mathrm{Hb}$ level in the feces (Figure $3 \mathrm{~b}$ ). At autopsy, their cecum and colon were swollen and infiltrated with blood. Such an appearance was never seen for Vanin-1/- mice, which had less abundant and more transient intestinal bleeding than WT mice. Typical intestinal samples from the two groups are displayed in Figure 3c. Histological and immunohistological analysis of colon cryosections performed 8 weeks after infection showed a severe disorganization of the mucous membrane of the colon in WT but not Vanin-1/- mice. In WT mice, crypts of Lieberkühn were reduced in depth, with increased diameter and more mucus production (Figure 4), compared with the Vanin-1/- mice that presented histologically normal crypts of Lieberkühn. In WT mice, peroxidase-positive cells were detected both in the submucosa and in the lamina propria of mucous membrane between the crypts of Lieberkühn, whereas these cells remained confined to the submucosa in Vanin-1/- mice. Confocal analysis shows that peroxidase-positive cells were in close contact with disrupted Ep-CAM-positive epithelial lining of the crypts of Lieberkühn in WT mice. In contrast, in Vanin-1/- mice myeloid cells remained in the submucosa. This submucosal localization was noticeable even in granulomatous areas around parasite eggs in Vanin-1/- mice. Taken as a whole, myeloid cell infiltration was quantified by measuring the percentage of the total area covered by peroxidase-positive cells on tissue sections of colons (WT $=3.2 \%$ versus Vanin $-1^{-/}$ $=1.3 \% ; P<0.01)$ and by the global MPO activity extracted from whole tissues ( $\mathrm{WT}=8.5 \pm 1.2$ versus Vanin-1/- $=6.4 \pm 0.5 \mathrm{mU} / \mathrm{mg}$ protein; $P<0.01$ ). All these findings show that myeloid cell infiltration and tissue damage were more pronounced in WT than in Vanin-1/-infected mice.

Lack of Vanin-1 led to increased GSH levels in liver and intestine. In the liver, GSH stores were significantly higher in Vanin-1 $1^{-/}$than in WT mice in either healthy or experimental animals (Table 1a). Changes in GSH levels reflected changes in $\gamma$-GCS activity, which was, in all cases, significantly higher in Vanin-1//- than in WT mice, whereas GSH reductase (GR) activity was comparable (56.7 \pm 3.1 and $48.7 \pm 2.6 \mathrm{mU} / \mathrm{mg}$ protein in $\mathrm{WT}$ and Vanin-1/- mice, respectively). S. mansoni infection triggered a major oxidative stress in WT mice; consequently, liver GSH levels dropped dramatically despite an increase in $\gamma$-GCS enzyme activity. GR activity was also reduced to $29.5 \pm 3.8 \mathrm{mU} / \mathrm{mg}$ protein. Strikingly, in Vanin-1 $1^{-/}$mice infection induced a drastic increase in $\gamma$-GCS activity, which was significantly higher than in WT mice, whereas GR activity remained unchanged, suggesting a better preservation of GSH homeostasis in this group. As a result, GSH levels did not show the decrease observed in WT animals. Upon indomethacin treatment, similar variations in $\gamma$-GCS activity were observed. In this case, GSH stores were maintained at normal levels in WT mice whereas they were enhanced in Vanin-1/- mice. The oxidized glutathione/GSH ratio was less than $1 \%$ in 
a

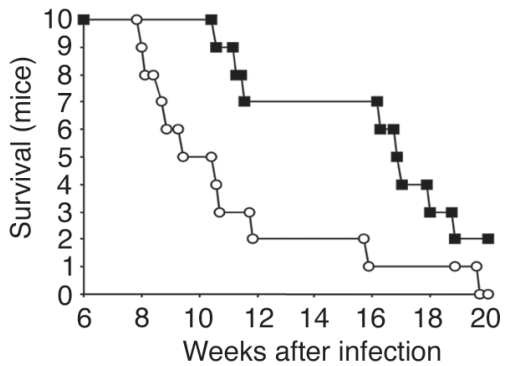

b

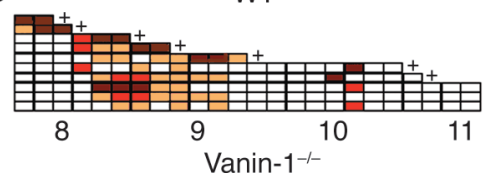

Vanin-1-1-

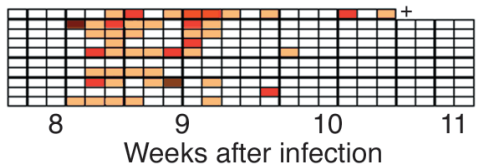

$\square<35$

$\square 35-120$

口 120-300 $\mathrm{Hb} / \mathrm{mg}$ excrement

$$
\square>300
$$

untreated mice and less than $2 \%$ in inflamed mice (data not shown). Importantly, cystamine administration dramatically reduced both $\gamma$-GCS activity and GSH levels in control mice as well as in indomethacin-treated animals. As a result, cystamine suppressed the difference between Vanin-1 $1^{-/-}$and WT mice.

In the intestine, GSH concentrations paralleled those observed in the liver, however, at a lower level: GSH stores were significantly higher in the absence of Vanin-1 in untreated as well as in indomethacin-treated mice. Basal $\gamma$-GCS activity needed for constant renewal of GSH intestinal pools from liver-derived GSH was identical in both types of mice. In contrast, indomethacin treatment induced a significant reduction in $\gamma$-GCS activity in WT mice whereas activity remained unchanged in Vanin-1 $1^{-/}$mice. As observed in the liver, cystamine administration annihilated the effects of Vanin-1 inactivation; therefore, it reduced intestinal GSH levels in both groups of mice.

Under our experimental conditions, changes in $\gamma$-GCS activity were not associated with changes in enzyme expression: in liver, as in intestine, Vanin $-1^{-/-}$mice dis-

\section{Figure 3}

Prolonged survival of S. mansoni-infected Vanin-1/- mice. (a) Survival curves of Vanin-1/- (square) and WT (circle) mice infected with a lethal dose of S. mansoni cercariae. From 8 to 12 weeks after infection, hemoglobin amount in feces was assayed daily. (b) Reduced intestinal hemorrhages in Vanin-1/- mice following S. mansoni infection. Daily quantification of the $\mathrm{Hb}$ amount in infected Vanin-1/- and WT mice feces. Color scale indicates $\mathrm{Hb}$ amount, namely less than 35 , 35-120, 120-300, and more than $300 \mathrm{ng} \mathrm{Hb} / \mathrm{mg}$ excrement. +, dead mice. (c) Typical aspect of cecum and colon of WT mice (top) and Vanin-1 $1^{-/}$mice (bottom). Scale bars, $1 \mathrm{~cm}$.

played normal levels of $\gamma$-GCS catalytic and modifier subunit mRNAs. The latter remained unchanged after indomethacin treatment (data not shown).

\section{Discussion}

We showed that Vanin-1/- mice are less susceptible to intestinal inflammation, either acute (indomethacin treatment) or chronic (S. mansoni infection). In the $S$. mansoni infection model, a main pathological feature is the presence of diffuse intestinal hemorrhages (40). Such injuries were dramatically reduced in the absence of Vanin-1, with consecutive prolonged survival after lethal infection. WT and Vanin-1/- mice had comparable parasite burdens, since equivalent numbers of eggs were found in both groups (data not shown). Vanin-1-deficient mice that survived intestinal injury later died from liver disease, since disease progression in the liver proceeded similarly in Vanin$1^{-/-}$and WT mice (data not shown). This discrepancy between liver and intestinal effects of the lack of Vanin-1 could be related to the distinctive form of chronic liver inflammation induced by $S$. mansoni, since the inflammatory reaction protects liver against the cytotoxic compounds released by the eggs (41). The positive effects of attenuated inflammatory response are counterbalanced by a prolonged survival of parasite eggs in the organ (our unpublished data).

In the indomethacin inflammatory model, several transcripts associated with inflammatory cell activation known to be induced by this drug $(42,43)$ were barely expressed or even undetectable in the intestine of Vanin-1-/ mice. Among them is MIP-2, a local chemoattractant for neutrophils (44), which plays an important role in the progression of indomethacin-induced intes-
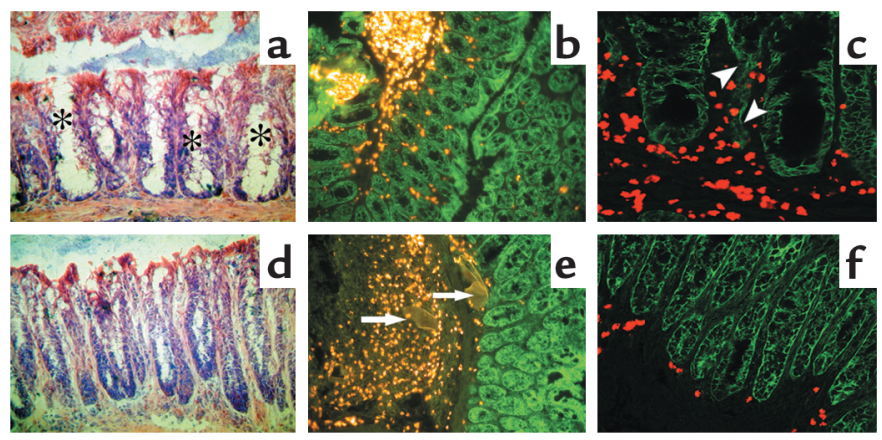

\section{Figure 4}

Cryosections of colon $(\times 100)$ from three, independent, infected WT $(\mathbf{a}-\mathbf{c})$ or Vanin-1/- $(\mathbf{d}-\mathbf{f})$ mice at 8 weeks after infection. Sections were stained with $\mathrm{H} \& \mathrm{E}$ ( $\mathbf{a}$ and $\mathbf{d}$ ) or stained with tyramideCy3 (b-e), a peroxidase substrate (yellow/red fluorescence) combined with anti-Ep-CAM (green fluorescence) mAb. Note increased mucus production in the crypts of Lieberkühn $\left({ }^{*}\right)$ in WT mice. These pictures are representative of images observed in independent WT $(n=6)$ or Vanin-1/- $(n=8)$ mice, and quantification is performed using the Metamorph program as described in the text. Arrowheads indicate disrupted intestinal mucosa; arrows indicate Schistosoma eggs. 


\section{Table 1}

Enhanced $\gamma$-GCS activity and GSH levels in the liver and intestine of indomethacin-treated and S. mansoni-infected WT and Vanin-1/- mice

\begin{tabular}{lcccc}
\hline & \multicolumn{2}{c}{ WT } & \multicolumn{2}{c}{ Vanin-1 $^{-/-}$} \\
& GSH & $\gamma$-GCS & GSH & $\gamma$-GCS \\
Liver & & & & \\
Controls & $8.56 \pm 1.8$ & $16.5 \pm 2.2$ & $12.7 \pm 2.4^{\mathrm{A}}$ & $23.9 \pm 3.4^{\mathrm{A}}$ \\
Cystamine & $5.36 \pm 1.1$ & $10.8 \pm 0.6$ & $5.55 \pm 1.2$ & $11.2 \pm 0.5$ \\
Indomethacin & $9.50 \pm 1.4$ & $23.4 \pm 0.7$ & $16.6 \pm 1.2^{\mathrm{A}}$ & $37.9 \pm 3.3^{\mathrm{A}}$ \\
Indomethacin + cystamine & $7.60 \pm 0.6$ & $19.0 \pm 4.1$ & $8.30 \pm 0.9$ & $21.6 \pm 1.7$ \\
Infection with S. mansoni & $3.96 \pm 1.8$ & $20.6 \pm 2.8$ & $11.09 \pm 1.8^{\mathrm{A}}$ & $37.2 \pm 2.3^{\mathrm{A}}$ \\
Intestine & & & & \\
Controls & $1.03 \pm 0.1$ & $26.5 \pm 4.2$ & $1.84 \pm 0.2^{\mathrm{A}}$ & $23.6 \pm 4.5$ \\
Cystamine & $0.90 \pm 0.1$ & $20.2 \pm 1.2$ & $1.0 \pm 0.1$ & $21.5 \pm 2.4$ \\
Indomethacin & $0.56 \pm 0.1$ & $18.4 \pm 2.2$ & $1.37 \pm 0.2^{\mathrm{A}}$ & $23.7 \pm 2.6^{\mathrm{A}}$ \\
Indomethacin + cystamine & $0.65 \pm 0.1$ & $17.7 \pm 1.4$ & $0.75 \pm 0.1$ & $20.6 \pm 1.0$
\end{tabular}

Cystamine was administered to compensate for Vanin-1 inactivation. GSH levels and $\gamma$-GCS activity are expressed in micromoles per gram of tissue and milliunits per milligram of protein, respectively. AValues significantly different from the WT group $(P<0.05$, Student $t$ test $)$. tion is not absolute between the two pantetheinase isoforms. This result is in agreement with the fact that cysteamine production is undetectable in the kidney and liver of Vanin-1/- mice (6). Vanin-3 is probably secreted and might exert its effects at distant sites (4).

In vitro studies show that cystamine downregulates $\gamma$-GCS activity, acting as a glutamine analogue (17-19). Our findings show that similar effects are observed in vivo. Undetectable pantetheinase activity and cysteamine levels in Vanin-1-/mice were associated with higher $\gamma$-GCS activity in the liver, as compared with WT mice, either in control or experimental animals. In agreement, cystamine administration dramatically reduced the

tinal inflammation (24). Absence of MIP-2 expression in Vanin-1-/- mice was, as expected, associated with a reduced MPO activity. In agreement, in humans the migratory function of neutrophils involves the Vanin cluster (45).

Protection of Vanin-1/- mice following indomethacin treatment was associated with higher GSH levels, as compared with WT animals. GSH is well known to play crucial regulatory functions in the context of inflammation because it is required to maintain the cellular redox status and to scavenge free radicals. Furthermore, GSH modulates immune functions by the regulation of several pathways, including lipid mediator synthesis as a cofactor of glutathione peroxidases $(46,47)$. Increase in GSH levels in Vanin-1/- mice likely results from enhanced liver synthesis as indicated by an increase in $\gamma$-GCS activity. Indeed, this organ serves as a central GSHgenerating organ that supplies kidney and intestine with other constituents of GSH resynthesis (48) through the catabolism of GSH by $\gamma$-glutamyltranspeptidase (49). As a consequence, GSH stores are lower in the intestine than in the liver (49-51). In agreement, we found that GSH levels in the intestine reflected GSH status in the liver, with values significantly higher in treated Vanin-1/- than in WT mice. Basal $\gamma$-GCS activity, which is required for the maintenance of a normal GSH pool in the intestine, was comparable in Vanin-1/- and WT mice. This suggests that under homeostatic conditions the intestinal GSH pool might be dependent mainly upon liver production through GSH metabolites. Under stressed conditions that enhance Vanin-1 expression (data not shown), however, $\gamma$-GCS activity drops in WT and not Vanin-1/- tissues, reflecting the local inhibitory effect of cysteamine production as occurs in the liver. Finally, despite the presence of Vanin-3 transcripts in the liver of Vanin-1-/- mice (data not shown), our results seem to suggest that compensa- enzyme activity and GSH levels in such a way that the difference between the two groups was cancelled. Cystamine action seems to mimic the negative feedback regulation of $\gamma$-GCS by GSH through the GSH binding site while interacting with a sulfhydryl group at a second site (52). Other disulfides are unable to fulfill these rather stringent requirements and therefore are unable to inhibit this enzyme (19). This provides a likely explanation for the gastrointestinal ulcerogenic effect of cysteamine (21).

Because of its pleiotropic effects, however, cystamine might exert a protective effect in some particular cases. Administration of low doses of cystamine is assumed to inhibit transglutaminase activity in the brain and decreases Huntington disease symptoms in the mouse (53).

Our results show that pantetheinase activity of Vanin-1 molecule is a major regulator of intestinal inflammation, acting through cysteamine release. This effect might be due to the combined effect of downregulation of GSH levels and enhanced recruitment of myeloid cells at inflamed sites; therefore, pantetheinase inhibitors would have protective effects against intestinal inflammatory disorders. These findings provide a rationale for a new anti-inflammatory strategy.

\section{Acknowledgments}

We thank S. Dupré, G. Pitari, A. Greenfield, L. Leserman, and J. Ewbank for reading the manuscript. This study was supported by institutional grants from the Institut National de la Santé et de la Recherche Médicale and the Centre National de la Recherche Scientifique, as well as charitable trusts from the Association pour la Recherche contre le Cancer (ARC 5945), the Imagerie pour le Petit Animal, and the Association F. Aupetit. F. Malergue, F. Martin, and M.F. Penet are recipients of a grant from the Ministère de l'Education Nationale, de la Recherche et de la Technologie, and the Ligue Nationale contre le Cancer. 
1. Aurrand-Lions, M., et al. 1996. Vanin-1, a novel GPI-linked perivascular molecule involved in thymus homing. Immunity. 5:391-405.

2. Granjeaud, S., Naquet, P., and Galland, F. 1999. An ESTs description of the new Vanin gene family conserved from fly to human. Immunogenetics. 49:964-972.

3. Galland, F., et al. 1998. Two human genes related to murine vanin-1 are located on the long arm of human chromosome 6. Genomics. 53:203-213.

4. Martin, F., et al. 2001. Vanin genes are clustered (human 6q22-24 and mouse 10A2B1) and encode isoforms of pantetheinase ectoenzymes. Immunogenetics. 53:296-306.

5. Maras, B., Barra, D., Dupre, S., and Pitari, G. 1999. Is pantetheinase the actual identity of mouse and human vanin-1 proteins? FEBS Lett. 461:149-152.

6. Pitari, G., et al. 2000. Pantetheinase activity of membrane-bound Vanin-1: lack of free cysteamine in tissues of Vanin-1 deficient mice. FEBS Lett. 483:149-154.

7. Dupre, S., Graziani, M.T., Rosei, M.A., Fabi, A., and Del Grosso, E. 1970 The enzymatic breakdown of pantethine to pantothenic acid and cystamine. Eur. J. Biochem. 16:571-578.

8. Miller, S.L., and Schlesinger, G. 1993. Prebiotic syntheses of vitamin coenzymes. I. Cysteamine and 2-mercaptoethanesulfonic acid (coenzyme M). J. Mol. Evol. 36:302-307.

9. Garcia, R.A., Hirschberger, L.L., and Stipanuk, M.H. 1988. Measurement of cyst(e)amine in physiological samples by high performance liquid chromatography. Anal. Biochem. 170:432-440.

10. Ida, S., Tanaka, Y., Ohkuma, S., and Kuriyama, K. 1984. Determination of cystamine by high-performance liquid chromatography. Anal. Biochem. 136:352-356.

11. Kataoka, H., Imamura, Y., Tanaka, H., and Makita, M. 1993. Determination of cysteamine and cystamine by gas chromatography with flame photometric detection. J. Pharm. Biomed. Anal. 11:963-969.

12. Ricci, G., Nardini, M., Chiaraluce, R., Dupre, S., and Cavallini, D. 1983. Detection and determination of cysteamine at the nanomole level. J. Appl. Biochem. 5:320-329.

13. Siefring, G.E., Jr., Apostol, A.B., Velasco, P.T., and Lorand, L. 1978. Enzymatic basis for the $\mathrm{Ca} 2+$-induced cross-linking of membrane proteins in intact human erythrocytes. Biochemistry. 17:2598-2604.

14. Pontremoli, S., Traniello, S., Enser, M., Shapiro, S., and Horecker, B.L. 1967. Regulation of fructose diphosphatase activity by disulfide exchange. Proc. Natl. Acad. Sci. U. S. A. 58:286-293.

15. Namboodiri, M.A., Weller, J.L., and Klein, D.C. 1980. Rapid and reversible activation of acetyl CoA hydrolase in intact pineal cells by disulfide exchange. Biochem. Biophys. Res. Commun. 96:188-195.

16. Ernest, M.J., and Kim, K.H. 1973. Regulation of rat liver glycogen synthetase. Reversible inactivation of glycogen synthetase D by sulfhydryldisulfide exchange. J. Biol. Chem. 248:1550-1555.

17. Beamer, R.L., Griffith, O.W., Gass, J.D., Anderson, M.E., and Meister, A 1980. Interaction of L- and D-3-amino-1-chloro-2-pentanone with gamma-glutamylcysteine synthetase. J. Biol. Chem. 255:11732-11736.

18. Lebo, R.V., and Kredich, N.M. 1978. Inactivation of human gamma-glutamylcysteine synthetase by cystamine. Demonstration and quantification of enzyme-ligand complexes. J. Biol. Chem. 253:2615-2623.

19. Griffith, O.W., Larsson, A., and Meister, A. 1977. Inhibition of gammaglutamylcysteine synthetase by cystamine: an approach to a therapy of 5-oxoprolinuria (pyroglutamic aciduria). Biochem. Biophys. Res. Commun 79:919-925.

20. Griffith, O.W., and Mulcahy, R.T. 1999. The enzymes of glutathione synthesis: gamma-glutamylcysteine synthetase. Adv. Enzymol. Relat. Areas Mol. Biol. 73:209-267.

21. Troyer, K.L., et al. 2001. Growth retardation, duodenal lesions, and aberrant ileum architecture in triple null mice lacking EGF, amphiregulin, and TGF-alpha. Gastroenterology. 121:68-78.

22. Dessein, A.J., et al. 1999. Severe hepatic fibrosis in Schistosoma mansoni infection is controlled by a major locus that is closely linked to the interferon-gamma receptor gene. Am. J. Hum. Genet. 65:709-721.

23. Lambertucci, J.R., et al. 2000. Schistosoma mansoni: assessment of morbidity before and after control. Acta Trop. 77:101-109.

24. Beck, P.L., et al. 2000. Mechanisms of NSAID-induced gastrointestinal injury defined using mutant mice. Gastroenterology. 119:699-705.

25. Abdallahi, O.M., Hanna, S., De Reggi, M., and Gharib, B. 1999. Visualization of oxygen radical production in mouse liver in response to infection with Schistosoma mansoni. Liver. 19:495-500

26. Gharib, B., Abdallahi, O.M., Dessein, H., and De Reggi, M. 1999. Development of eosinophil peroxidase activity and concomitant alteration of the antioxidant defenses in the liver of mice infected with Schistosoma mansoni. J. Hepatol. 30:594-602.
27. Pascal, M., et al. 2000. Hyaluronate levels and markers of oxidative stres in the serum of Sudanese subjects at risk of infection with Schistosoma mansoni. Trans. R. Soc. Trop. Med. Hyg. 94:66-70.

28. La Flamme, A.C., Patton, E.A., Bauman, B., and Pearce, E.J. 2001. IL-4 plays a crucial role in regulating oxidative damage in the liver during schistosomiasis. J. Immunol. 166:1903-1911.

29. Wynn, T.A., et al. 1998. IL-10 regulates liver pathology in acute murine Schistosomiasis mansoni but is not required for immune down-modulation of chronic disease. J. Immunol. 160:4473-4480.

30. Naspetti, M., et al. 2000. A novel anti-Ep-CAM antibody to analyze the organization of thymic medulla in autoimmunity. Curr. Top. Microbiol. Immunol. 251:109-117.

31. Liaudet, L., et al. 2000. Protection against hemorrhagic shock in mice genetically deficient in poly(ADP-ribose)polymerase. Proc. Natl. Acad. Sci. U. S. A. 97:10203-10208.

32. Lyons, C.R., Orloff, G.J., and Cunningham, J.M. 1992. Molecular cloning and functional expression of an inducible nitric oxide synthase from a murine macrophage cell line. J. Biol. Chem. 267:6370-6374.

33. Ballou, L.R., Botting, R.M., Goorha, S., Zhang, J., and Vane, J.R. 2000 Nociception in cyclooxygenase isozyme-deficient mice. Proc. Natl. Acad. Sci. U. S. A. 97:10272-10276.

34. Scott, D.J., et al. 2001. Lack of inducible nitric oxide synthase promotes intestinal tumorigenesis in the $\mathrm{Apc}(\mathrm{Min} /+)$ mouse. Gastroenterology. 121:889-899.

35. Neumann, B., et al. 1999. Mechanisms of acute inflammatory lung injury induced by abdominal sepsis. Int. Immunol. 11:217-227.

36. Konecki, D.S., Brennand, J., Fuscoe, J.C., Caskey, C.T., and Chinault, A.C. 1982. Hypoxanthine-guanine phosphoribosyltransferase genes of mouse and Chinese hamster: construction and sequence analysis of cDNA recombinants. Nucleic Acids Res. 10:6763-6775.

37. Kinoshita, T., Imamura, J., Nagai, H., and Shimotohno, K. 1992. Quantification of gene expression over a wide range by the polymerase chain reaction. Anal. Biochem. 206:231-235

38. Seelig, G.F., and Meister, A. 1984. Gamma-glutamylcysteine synthetase from erythrocytes. Anal. Biochem. 141:510-514.

39. Tietze, F. 1969. Enzymic method for quantitative determination of nanogram amounts of total and oxidized glutathione: applications to mammalian blood and other tissues. Anal. Biochem. 27:502-522.

40. Cheever, A.W. 1985. Schistosoma japonicum: the pathology of experimental infection. Exp. Parasitol. 59:1-11.

41. Amiri, P., et al. 1992. Tumour necrosis factor alpha restores granulomas and induces parasite egg-laying in schistosome-infected SCID mice. Nature. 356:604-607.

42. Wallace, J.L., et al. 1998. Cyclooxygenase 1 contributes to inflammatory responses in rats and mice: implications for gastrointestinal toxicity. Gastroenterology. 115:101-109.

43. Tanaka, A., Araki, H., Hase, S., Komoike, Y., and Takeuchi, K. 2002. Upregulation of COX-2 by inhibition of COX-1 in the rat: a key to NSAIDinduced gastric injury. Aliment. Pharmacol. Ther. 16:90-101.

44. Wolpe, S.D., and Cerami, A. 1989. Macrophage inflammatory proteins 1 and 2: members of a novel superfamily of cytokines. FASEB J. 3:2565-2573

45. Suzuki, K., et al. 1999. A novel glycosylphosphatidyl inositol-anchored protein on human leukocytes: a possible role for regulation of neutrophil adherence and migration. J. Immunol. 162:4277-4284.

46. Lu, S.C. 1999. Regulation of hepatic glutathione synthesis: current concepts and controversies. FASEB J. 13:1169-1183.

47. Will, Y., et al. 2000. Gamma-glutamyltranspeptidase-deficient knockout mice as a model to study the relationship between glutathione status, mitochondrial function, and cellular function. Hepatology. 32:740-749.

48. Rana, S.V., Allen, T., and Singh, R. 2002. Inevitable glutathione, then and now. Indian J. Exp. Biol. 40:706-716.

49. Lieberman, M.W., et al. 1996. Growth retardation and cysteine deficiency in gamma-glutamyl transpeptidase-deficient mice. Proc. Natl. Acad. Sci. U. S. A. 93:7923-7926.

50. LeGrand, T.S., and Aw, T.Y. 1996. Chronic hypoxia and glutathionedependent detoxication in rat small intestine. Am. J. Physiol. 270:G725-G729.

51. Griffith, O.W., and Meister, A. 1979. Glutathione: interorgan translocation, turnover, and metabolism. Proc. Natl. Acad. Sci. U. S. A. 76:5606-5610.

52. Huang, C.S., Moore, W.R., and Meister, A. 1988. On the active site thiol of gamma-glutamylcysteine synthetase: relationships to catalysis, inhibition, and regulation. Proc. Natl. Acad. Sci. U. S. A. 85:2464-2468.

53. Karpuj, M.V., et al. 2002. Prolonged survival and decreased abnormal movements in transgenic model of Huntington disease, with administration of the transglutaminase inhibitor cystamine. Nat. Med. 8:143-149 